\title{
FATORES ASSOCIADOS À AUTOMEDICAÇÃO EM UMA FARMÁCIA COMUNITÁRIA DE OURO PRETO DO OESTE, RONDÔNIA
}

\author{
Juarez Marcos ARRABAL Júnior \& Jeferson de Oliveira SALVI*
}

Centro Universitário Luterano de Ji-Paraná. Ji-Paraná, RONDÔNIA, Brasil.

*Autor para correspondência: jefersonsalvi@hotmail.com

DOI: http://dx.doi.org/10.18571/acbm.177

\section{RESUMO}

O presente estudo teve o intuito de investigar os fatores associados à automedicação em uma farmácia comunitária em Ouro Preto do Oeste, Rondônia. Caracterizou-se os aspectos farmacológicos das principais classes de medicamentos identificadas e a relação com o automedicar-se. A pesquisa ocorreu por meio do levantamento de dados, de modo transversal e exploratório. A grande maioria declarou a prática da automedicação, havendo predominância entre os 18 e 28 anos, com renda de 1 a 2 salários mínimos. Observou-se uma associação significativa da prática entre os homens $(\mathrm{p}<0,001)$, sendo que, o risco relativo e a chance da automedicação foram bem maiores acima dos 49 anos. A classe terapêutica mais observada foi a dos analgésicos (90\%) seguida pelos anti-inflamatórios (65\%). Dentre os principais fatores associados à autoadministração se destacaram a facilidade do acesso aos medicamentos e a dificuldade de acesso à consulta médica. O principal risco declarado foi o de ocorrer alergias, mas a possibilidade de óbitos também foi percebida. Conclui-se sobre a necessidade de intervenções junto aos profissionais de saúde para que sejam prestadas informações que contribuam para a adesão à farmacoterapia, para o uso racional de medicamentos e para a melhora da qualidade de vida dos pacientes.

Palavras-chave: Hábitos de Consumo de Medicamentos; Polifarmácia; Uso Racional de Medicamentos; Automedicação racional.

\begin{abstract}
The present study aimed to investigate the factors associated with self-medication in clients of a community pharmacy of Ouro Preto do Oeste's tourist resort, in Rondônia state. It aimed to characterize the pharmacological aspects of the main classes of drugs identified, as well to relate about the self-medicating. A research carried out through data collection, in a transversal and exploratory study. The great majority practices the self-medication, predominating the age group between 18 and 28 years with income of 1 to 2 minimum wages. There was a significant association between men and self-medication ( $\mathrm{p}<0.001)$, with the relative risk and the chance of being self-medicated higher for men over 49 years. The most common class of medication used was the analgesics in $90 \%$ of participants, followed by anti-inflammatory drugs by about $65 \%$. Among the main factors associated with self-administration of drugs, it was highlighted the easily of access to medicines and the difficulty of access to medical consultation. Allergies was the risk declared, but the possibility of death also was perceived. In this way, we conclude about the accomplishment of a labor that praises the pharmacological treatment, for the rational use of medicines and for an improvement of the quality of life of the patients.
\end{abstract}

Keywords: Medication Consumption Habits; Polypharmacy; Rational Use of Medications; Rational self-medication. 


\section{Introdução}

O hábito de se automedicar pode ser compreendido como sendo a busca de um ou mais princípios ativos, tendo em vista a terapêutica de uma determinada patologia, sem o auxílio de profissionais habilitados (MONTASTRUC, 2016). A automedicação é uma prática comum, de difícil controle, que geralmente representa risco para a saúde da população em todo o mundo (LOPES et al., 2014). Essa prática, que se enquadra como autocuidado, pode ser definida como o uso de medicamentos por conta própria na intenção de tratar sinais e sintomas de doenças autodiagnosticadas (SCHMID et al., 2010).

No Brasil, estima-se que cerca de $35 \%$ dos medicamentos dispensados são utilizados como automedicação, envolvendo, em especial, os fármacos classificados como isentos de prescrição. Em 2015, a prática de automedicar-se foi determinada para aproximados $16 \%$ da população brasileira, sendo que, para a região Norte, a prevalência foi de 18\% (AQUINO et al., 2010; ARRAIS et al., 2015).

Dentre os fatores que contribuem para o seu aumento, está o excesso de propagandas de medicamentos, a própria facilidade da aquisição dos princípios ativos e a falta de conhecimento sobre os riscos (GALATO et al., 2012). De acordo com Cerqueira e colaboradores (2005), o fato de a farmácia ser considerada apenas um ponto comercial de vendas de medicamentos e correlatos, e não apenas um estabelecimento de saúde, é outro agravante que propicia a disseminação da automedicação. Outro fator importante a ser considerado é o acúmulo de medicamentos que parte da população mantém em suas residências, caracterizando a polifarmácia, formando, assim, um arsenal terapêutico de fácil acesso (FERREIRA et al., 2005).

O uso irracional dos medicamentos tornou-se um grave problema de saúde pública. Porém, mesmo diante das consequências, os países não procuram investir em políticas que objetivem promover o uso correto. Estima-se que mais de 50\% dos medicamentos são prescritos, dispensados ou vendidos de forma inapropriada (WHO, 2010).

A portaria 3.916/98 do ministério da saúde estabelece a Política Nacional de Medicamentos, que objetiva a eficácia, a segurança e a qualidade, bem como o acesso da população àqueles considerados essenciais, buscando, ainda, a promoção do uso racional (BRASIL, 1998). Para Silva e colaboradores (2012), mesmo diante das discussões sobre a automedicação, pouco se conseguiu, pois ela ainda se configura como um grave problema de saúde pública, atingindo todas as esferas sociais.

Diante do exposto, acredita-se na necessidade da adoção de medidas de conscientização dos profissionais que atuam diariamente com medicamentos, ainda durante a graduação, para que, quando atuantes no mercado de trabalho, eles sejam disseminadores de políticas de saúde que tenham como objetivo promover o uso racional de medicamentos, considerando, ainda, o melhor entendimento sobre conceitos a ele relacionado, tal como a polifarmácia (MASNOON et al., 2017).

O presente estudo teve o intuito de investigar os fatores associados à automedicação em clientes de uma farmácia comunitária na estância turística de Ouro Preto do Oeste, estado de Rondônia.

\section{Materiais e Métodos}

A pesquisa foi realizada em uma farmácia privada, de médio porte, localizada na Estância turística de Ouro Preto do Oeste, Estado de Rondônia (RO), após a aprovação pelo Comitê de Ética e Pesquisa em Seres Humanos do Centro Universitário Luterano de Ji-Paraná (CEPCEULJI/ULBRA), sob o número do parecer 2.552.795, de 20 de março de 2018. 


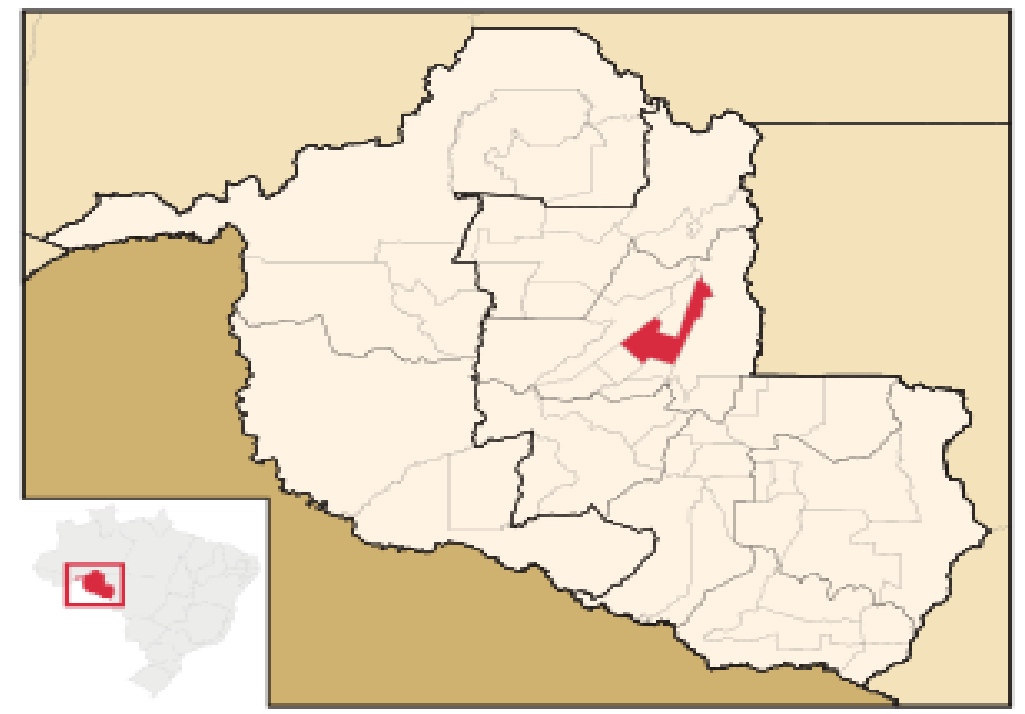

Figura 1: Localização geográfica da Estancia turística de Ouro Preto do Oeste, RO.

A estância turística está localizada na região Leste do estado de Rondônia e apresentou, no ano de 2017, uma população estimada de 39.759 habitantes (IBGE, 2018).

O estudo foi desenvolvido por meio do levantamento de dados qualiquantitativos, de modo transversal e exploratório, com aplicação de um questionário elaborado e aplicado aos clientes de forma aleatória, considerando-se como critério de inclusão a autodeclaração da prática de automedicar-se e a manutenção de polifarmácia.

Primeiramente, foi realizado o convite aos clientes da farmácia, de maneira aleatória e simples, por conveniência e, em seguida, explicou-se sobre o tema e os objetivos da pesquisa. Após o preenchimento do termo de consentimento livre e esclarecido (TCLE), foi realizada a entrega do questionário, contendo questões abrangendo dados pessoais, tais como: idade, sexo e renda familiar e perguntas específicas, considerando a investigação da prática da automedicação, classes medicamentosas, transtornos menores, dentre outros.

Os dados foram interpretados por meio de estatística descritiva percentual simples, tabulados e apresentados na forma de gráficos com auxílio do Microsoft Excel (2007 for Windows ${ }^{\circledR}$ ). Para a análise das associações, com auxílio do programa GraphPad Prism® (Versão 5.0), foi realizado o teste do Qui-quadrado, sendo analisadas a razão de chances e o risco relativo, os resultados foram considerados significativos para $\mathrm{p}<0,05$.

\section{Resultados e Discussão}

Em sua totalidade, foram aplicados 61 questionários, entre homens e mulheres, que possuíam idade igual ou superior a 18 anos. Destes, 55 declararam automedicarem-se.

A automedicação é uma das práticas responsáveis pelo desenvolvimento da resistência bacteriana aos diferentes antibióticos em todo o mundo. Considera-se que a privação de informações, bem como a qualidade delas, no ato da dispensação, pode levar à interrupção do tratamento quando do desaparecimento dos sintomas, por exemplo, favorecendo ainda mais a problemática (RATHER et al., 2017; JAMHOUR et al., 2017).

$\mathrm{Na}$ China, identificou-se uma alta prevalência do uso de antibióticos entre universitários, sendo que a grande maioria utilizava mais de um princípio ativo e uma parcela menor atribuiu efeito antiviral aos antibióticos (ZHU et al., 2016). No Brasil, o uso racional de antimicrobianos foi discutido e incentivado por políticas públicas, que estabeleceram, por meio da $\mathrm{RDC} \mathrm{n}^{\circ} 20$ de 2011 (BRASIL, 2011), a prescrição privativa para profissionais e estabelecimentos de saúde, tornando os princípios ativos sujeitos ao controle especial. 
Na figura 2, é possível observar a comparação entre o intervalo de faixas etárias e os sexos dos praticantes da automedicação. Constatou-se uma associação significativa entre os homens e o automedicar-se $(\mathrm{p}<0,001)$, sendo que o risco relativo e a chance da automedicação são bem maiores para os homens acima 49 anos (risco relativo $=1,3$; Odds ratio $=51,19$ ). Tal observação também foi encontrada por Moura e colaboradores (2014), que evidenciaram o maior percentual da automedicação em pessoas do sexo masculino em comparação às mulheres.

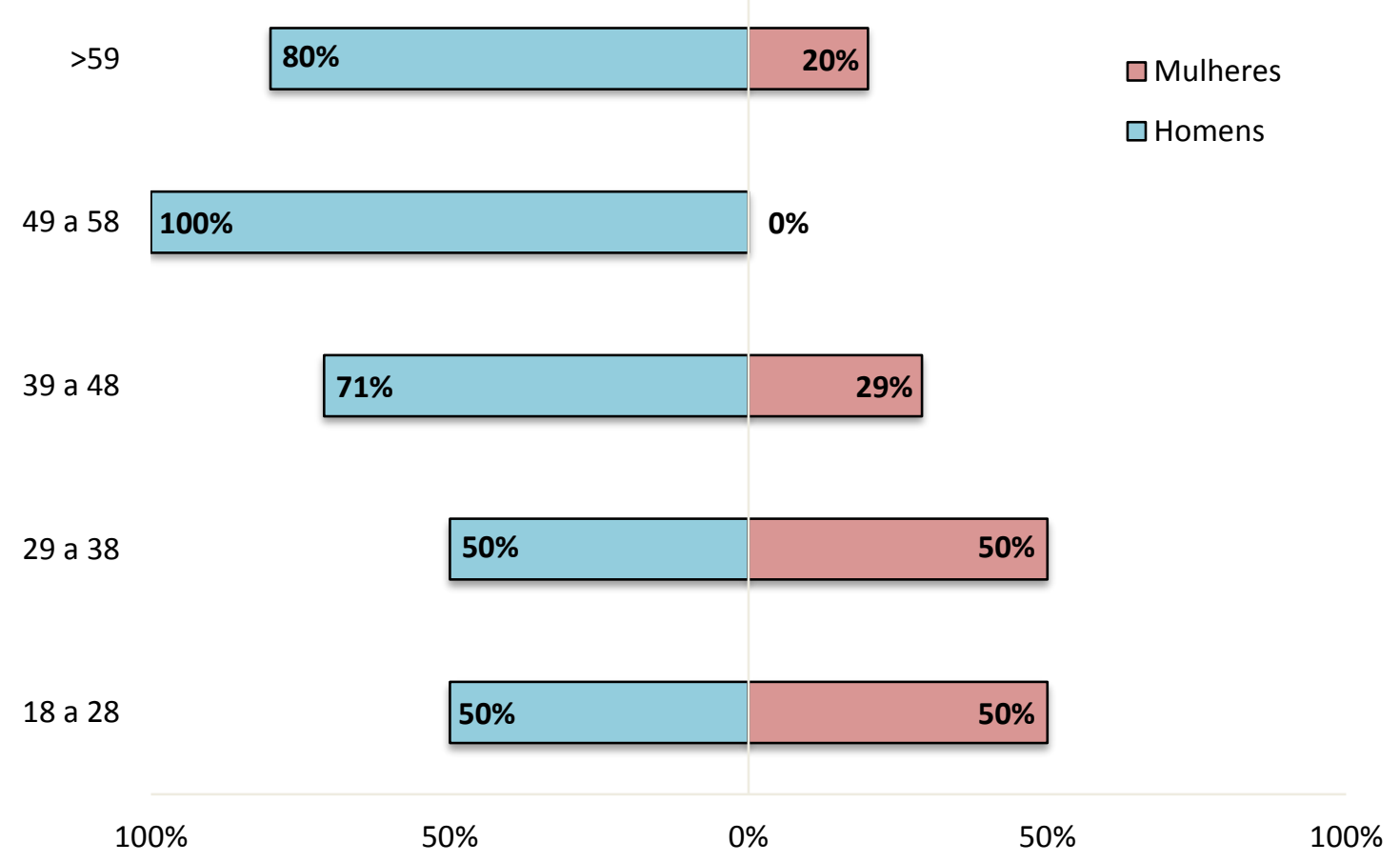

Figura 2: Pirâmide etária relacionada à distribuição dos sexos dos participantes da pesquisa realizada na estância turística de Ouro Preto do Oeste (RO). ${ }^{*} \mathrm{p}<0,001$, Teste Qui-quadrado.

Os princípios ativos foram classificados e organizados conforme demonstra a Figura 3, sendo que, um mesmo participante pode ter citado mais de um fármaco.

A classe dos analgésicos foi apontada por $92,72 \%$ dos entrevistados, seguida pela dos antiinflamatórios, mencionados por $64,28 \%$ dos entrevistados. Resultados semelhantes foram obtidos por Martines e colaboradores (2014), evidenciando a predominância do uso de analgésicos e antiinflamatórios na prática da automedicação para a dor muscular. Flaiti e pesquisadores (2014) também relataram a maior prevalência da automedicação, entre estudantes universitários, associada aos analgésicos.

Ressalta-se que a referência aos analgésicos se associa aos anti-inflamatórios atípicos, tais como: dipirona e paracetamol e estes, na realidade, são desprovidos ou apresentam pouco efeito anti-inflamatório, fazendo com que sejam mais indicados como antipiréticos e analgésicos. Por tanto, no estudo, considerou-se como anti-inflamatórios os princípios ativos não esteroidais (AINES) e esteroidais (AIES), em que tal atividade predomina, apesar de também exercer relativo efeito analgésico e antipirético. 


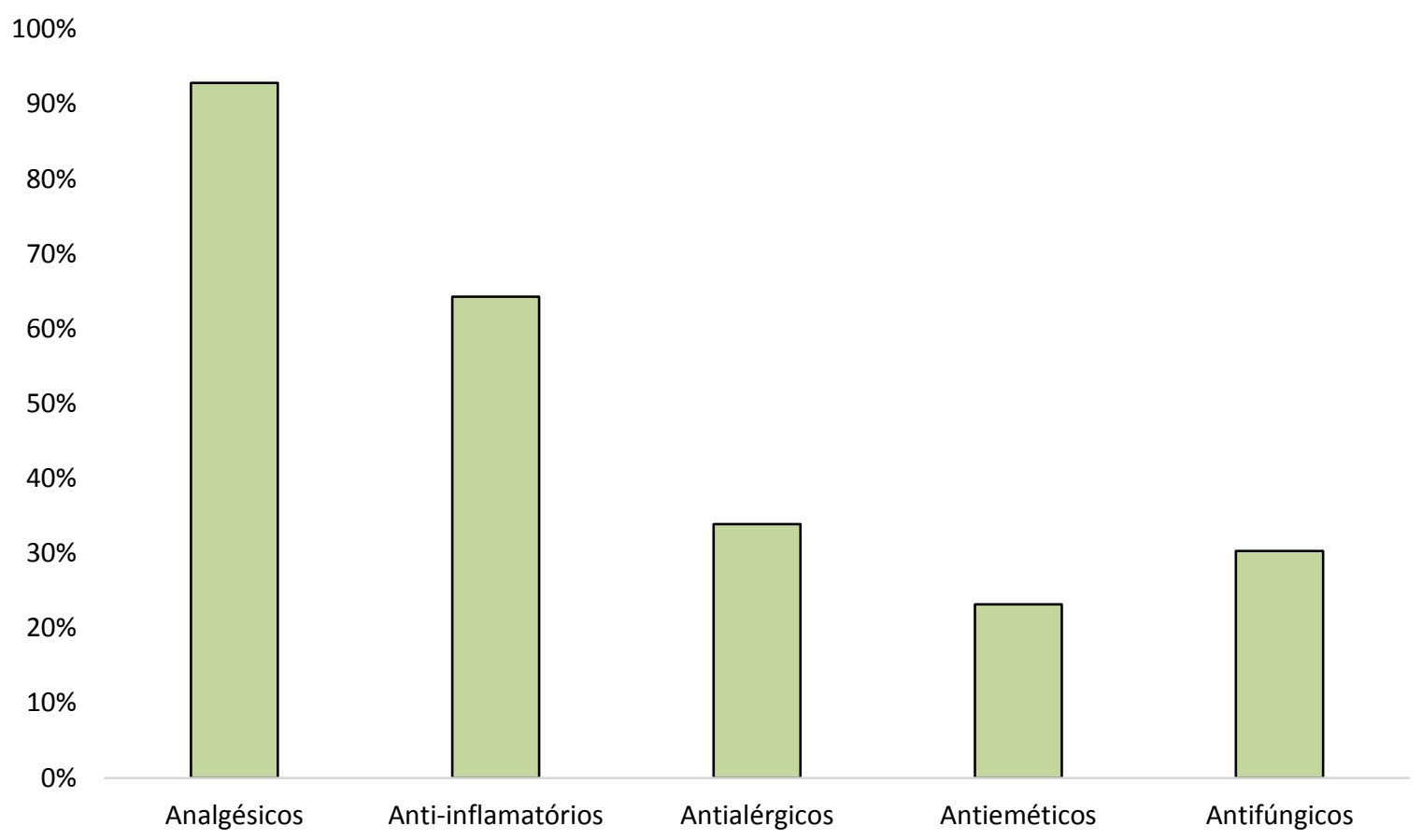

Figura 3. Distribuição das classes medicamentosas mais utilizadas na prática da automedicação dos participantes da pesquisa realizada na estância turística de Ouro Preto do Oeste (RO).

$\mathrm{Na}$ análise das principais queixas autodeclaradas, que incentivaram a prática da automedicação, identificou-se a dor, seguida pelos sintomas da gripe, alergia e problemas gastrointestinais, como os principais fatores associados à busca e à aquisição das classes medicamentosas anteriormente apresentadas, conforme demonstrado na Figura 4. Do mesmo modo que para os princípios ativos, um mesmo participantes pôde mencionar mais de uma causa.

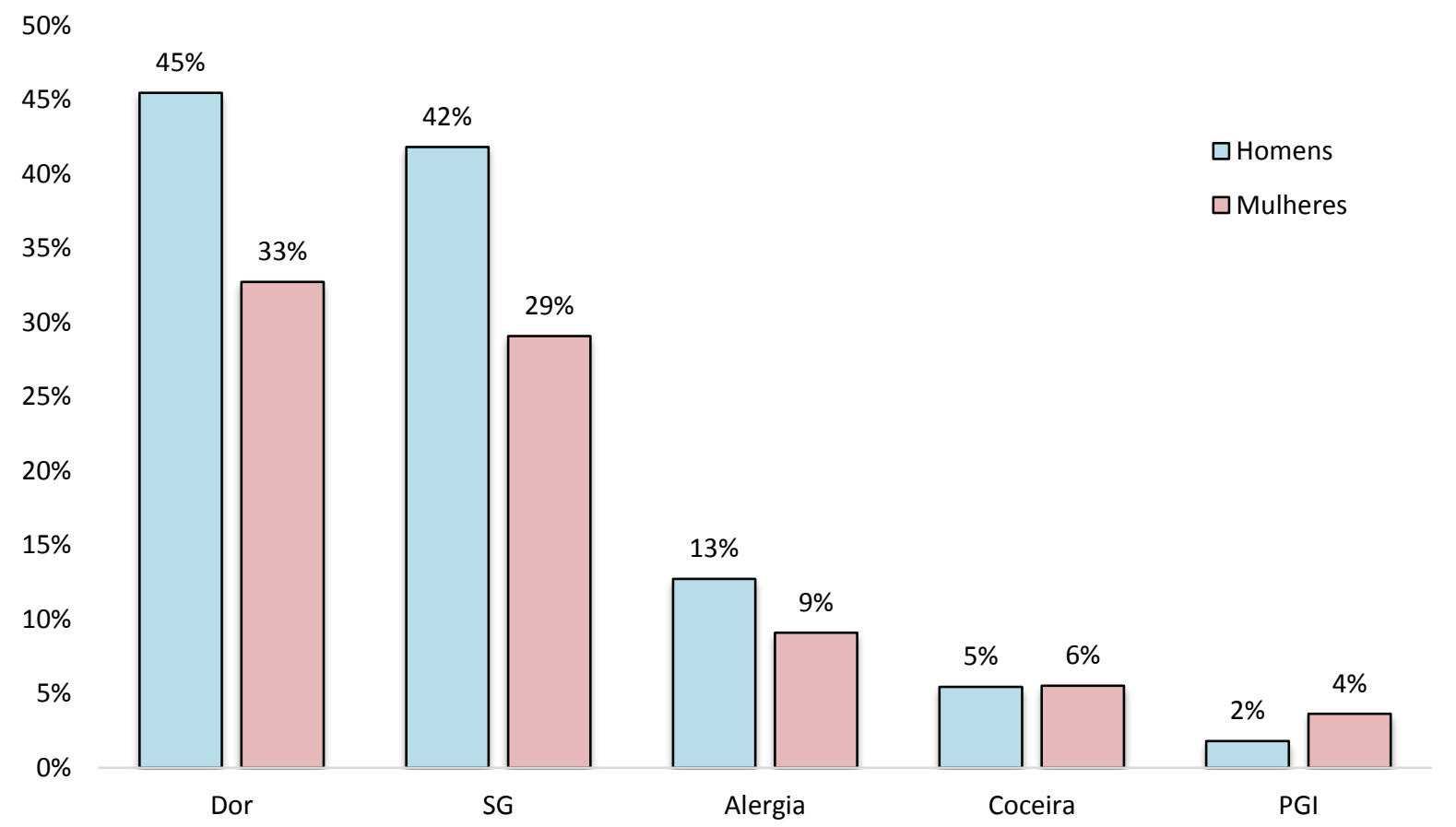

Figura 4. Distribuição dos transtornos menores associados ao sexo dos participantes da pesquisa realizada na estância turística de Ouro Preto do Oeste $(\mathrm{RO}) . \mathrm{SG}=$ sintomas da gripe, $\mathrm{PGI}=$ problemas gastrointestinais. 
Em estudo semelhante, Gama e colaboradores (2017) observaram que a dor foi o principal motivo associado ao automedicar-se e, do mesmo modo que os presentes resultados, os sintomas associados à gripe estiveram em segundo lugar. A justificativa para a predominância dessas classes medicamentosas pode encontrar explicação no fato de que a maioria dos princípios ativos utilizados são medicamentos isentos de prescrição (MIPs) e, geralmente, objetivam a terapêutica para transtornos menores (Monstatruc et al., 2016).

Dentre os fatores atribuídos à busca pela automedicação, estão: a facilidade de acesso ao medicamento, a dificuldade de acesso à consulta médica, bem como a falta de tempo para ir às consultas, a economia com gastos em consultas e, finalmente, alguns indivíduos consideraram possuir conhecimentos suficientes para se automedicar, conforme demonstra a Figura 5.

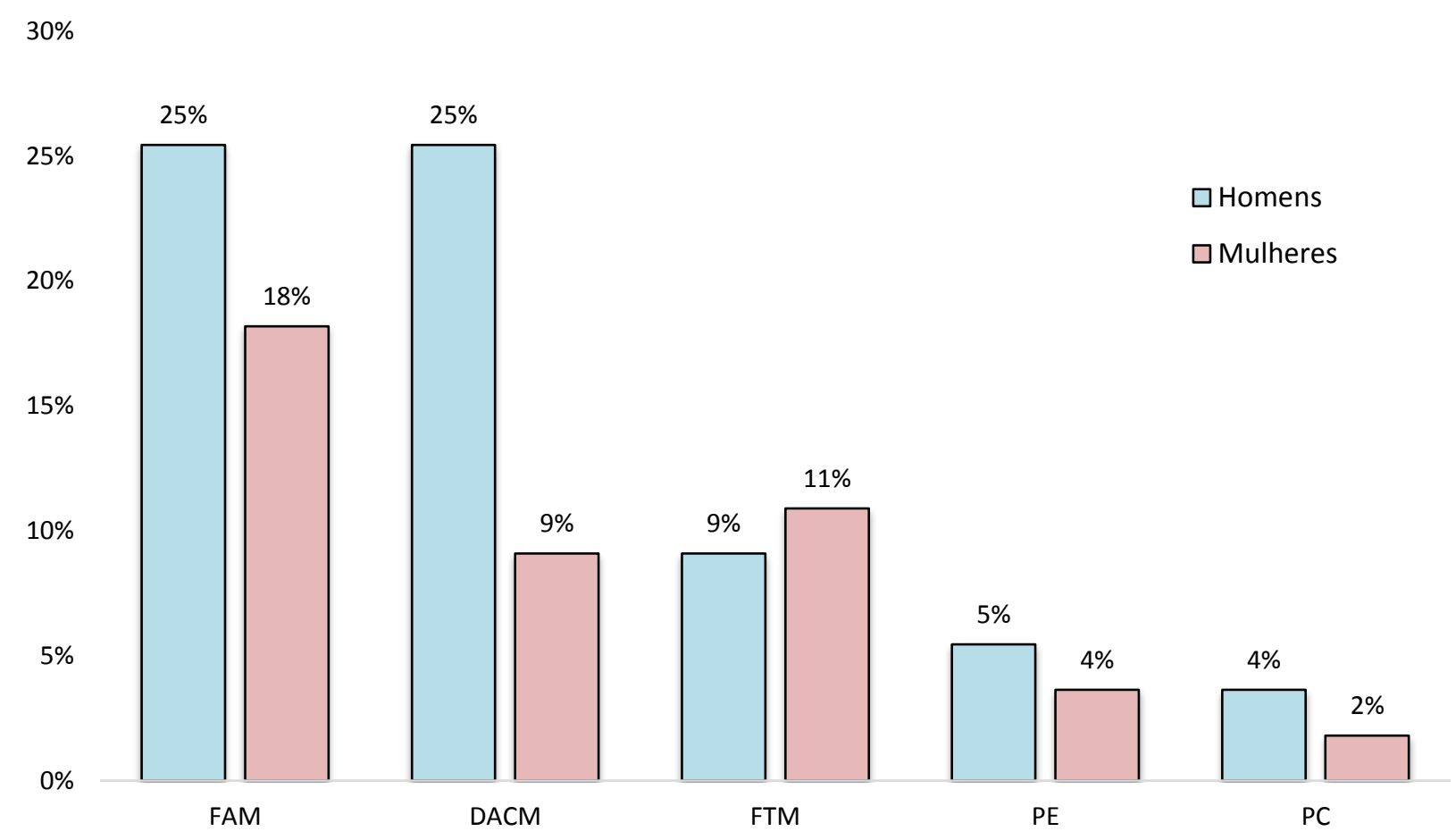

Figura 5. Distribuição dos fatores que levam ao hábito de se automedicar associados ao sexo dos participantes da pesquisa realizada na estância turística de Ouro Preto do Oeste (RO). FAM = facilidade de acesso ao medicamento, $\mathrm{DACM}=$ dificuldade de acesso à consulta médica, FTM = falta de tempo para ir ao médico, PE = para economizar, $\mathrm{PCS}=$ possui conhecimento suficiente para se automedicar.

Silva e colaboradores (2011) defendem que um dos principais motivos que leva a população à prática da automedicação é o alto custo das consultas médicas aliado à demora do Sistema Único de Saúde (SUS) em disponibilizar as consultas. Além disso, discute-se que a facilidade de acesso ao medicamento e à exploração massiva da publicidade contribui diretamente para a existência e para o aumento da automedicação em todo o mundo. Em alguns países, como a Sérvia, a exploração da mídia no processo de informação chega aos 40\% (TASIC et al., 2005; Aquino et al., 2010).

Nesse contexto, considera-se importante enfatizar que a prática da automedicação racional pode auxiliar no tratamento dos sinais e sintomas desses transtornos menores, considerando-se a redução dos atendimentos em locais onde a infraestrutura dos serviços da saúde não é adequada ou é insuficiente (Flaiti et al., 2014). Há evidências de que a orientação correta para a farmacoterapia pode contribuir para a melhora do quadro sintomatológico e para a própria descoberta da origem da doença. Por isso, no Brasil, o Farmacêutico junto à assistência em saúde conquistou seu espaço com a legalidade das suas atribuições clínicas, possiblidade de prescrições 


\section{Biomedica Brasiliensia}

dos MPIs e o reconhecimento das farmácias e drogarias como estabelecimentos de saúde (Brasil 2013a; Brasil 2013b; Brasil, 2014).

A Organização Mundial de Saúde (WHO, 2014) pondera que as indicações terapêuticas devem ser realizadas após diagnóstico prévio, para que o medicamento correto seja administrado de forma correta e não ocorra o prolongamento do sofrimento do paciente. Além disso, o autocuidado pode ser considerado necessário para a prevenção de doenças, sendo o principal recurso de qualquer sistema de saúde, podendo levar a mudanças no hábito de vida, tais como a cessação do tabagismo e da ingestão de bebidas alcoólicas (WHO, 1998).

A aquisição de medicamentos sem prescrição, especialmente para os princípios cuja legislação exige a retenção do receituário, é um problema relatado em todo o mundo. Alguns estudos identificam que esse modo de acesso ao medicamento, independente do sexo ou nível de instrução, está associado às experiências satisfatórias anteriores (FLAITI et al., 2014) e à falta de fiscalização por parte das entidades governamentais que, desse modo, contribuem para que produtos sejam vendidos em outros estabelecimentos e o atendimento seja feito por pessoas despreparadas, até mesmo em drogarias e farmácias (OLIVEIRA et al., 2013; CAVACO et al., 2017).

A presente pesquisa também investigou o conhecimento dos participantes sobre o risco de se automedicarem. Houve absoluta predominância para os sintomas alérgicos e as respostas foram padronizadas na Figura 5.

Considerando a linha tênue entre o autocuidado e os ricos inerentes ao uso irracional de medicamentos, a conscientização da existência do perigo agrava-se ainda mais quando da existência da polifarmácia. Há registro do aumento dessa prática nos últimos anos como recurso emergencial, e o que se pondera é que os diferentes princípios ativos administrados concomitantemente podem interagir entre si e desencadearem reações adversas fatais (RIBERIO et al., 2010; RAWLE et al., 2018).

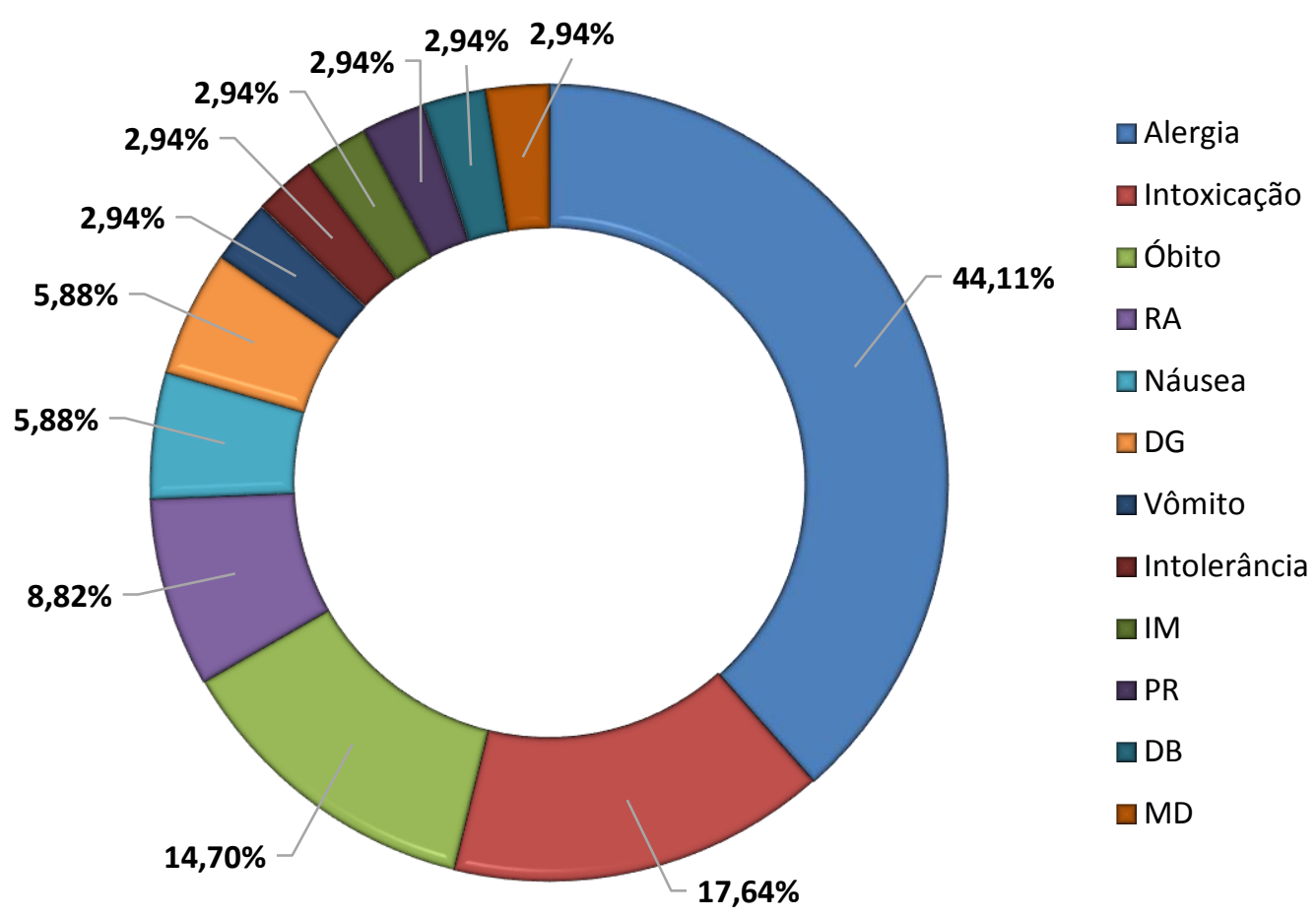

Figura 6. Distribuição dos riscos autodeclarados relacionados à prática da automedicação na estância turística de Ouro Preto do Oeste $(\mathrm{RO})$. *RA = reação adversa, $\mathrm{DG}=$ Dor gástrica, $\mathrm{IM}=$ interação medicamentosa, $\mathrm{PR}=$ problemas renais, $\mathrm{DB}=$ descritos na bula, $\mathrm{MD}=$ mascarar doenças. 
Presumindo que os participantes consideram os riscos aceitáveis e que são muitos os desdobramentos negativos associados à automedicação, sem menosprezar os seus benefícios, convém a discussão do termo automedicação racional, cujas principais características da sua origem estejam atreladas à necessidade de otimização do tempo para o início da farmacoterapia, a crença de se tratar de um transtorno menor e experiências anteriores positivas. Desse modo, a efetividade do seguimento farmacoterapêutico depende da presença atuante do profissional farmacêutico durante o ato da dispensação, uma vez que ele representa o contato mínimo ou a barreira que o paciente, com ou sem prescrição profissional, tem entre o produto e o início da sua utilização.

A automedicação racional, portanto, deve sofrer influência do farmacêutico no ato da dispensação como parte investigativa da assistência farmacêutica e de tomada de ações junto à promoção do uso racional dos medicamentos (ISRAEL et al., 2016).

\section{Considerações Finais}

A prática da automedicação esteve associada predominantemente ao sexo masculino, sobretudo entre os maiores de 49 anos, que buscaram analgésicos para o tratamento da dor. Dentre os fatores que incentivaram a aquisição dos medicamentos, destacaram-se a facilidade do acesso aos medicamentos e a dificuldade de acesso às consultas médicas. Entre o autoconhecimento dos riscos inerentes à prática de automedicar-se, reações alérgicas ocuparam o primeiro lugar.

Reflexionou-se sobre o entendimento e a necessidade da automedicação racional ao se ponderar sobre as vantagens e a possibilidade de medidas intervencionistas atribuídas ao profissional farmacêutico, que tem subsídios para a investigação dos problemas relacionados aos medicamentos, bem como na prestação de informações que contribuam para a adesão à farmacoterapia, para o uso racional de medicamentos e para a melhora da qualidade de vida dos pacientes.

\section{Referências}

AQUINO, D. S. de. BARROS, J. A. C. de. SILVA, M. D. P. da. A automedicação e os acadêmicos da área de saúde. Ciências \& Saúde Coletiva, Recife-PE, v. 15, n. 5, 2010. Brasil. Ministério da Saúde. Gabinete do Ministro. Portaria nº 3.916, de 30 de outubro de 1998. Aprova a Política Nacional de Medicamentos. Brasília: Diário Oficial da União; 1998. p.2.

ARRAIS, P.S.D et al. Revista de Saúde Pública. Revista de saúde publica, v. 50, p. 13s, 2016.

CERQUEIRA, G. S. et al. Perfil da automedicação em acadêmicos de enfermagem na cidade de João Pessoa. Conceitos. V.11/12, n.6, p.223-229, 2005.

CONSELHO FEDERAL DE FARMÁCIA. Resolução 585 e 586, e da lei 13.021 de 2014. Brasilia : 2013.

DE OLIVEIRA, R.I. B; GOMES, A.T; DA SILVA, D.A. Prática da automedicação por clientes de uma farmácia comunitária do município de MURIAÉ-MG. Acta Biomédica Brasilienses, v. 4, n. 2, p. 90-105, 2013.

FERREIRA, W.A. et al. Avaliação de farmácia caseira no município de Divinópolis (MG) por Estudantes do Curso de Farmácia da Unifenas. Revista Infarma, v, 17, n. 7/9, 2005. 
GALATO, D. MADALENA, J. PEREIRA, G. B. Automedicação em estudantes universitários: a influência da área de formação. Ciência \& Saúde Coletiva, v. 17, n. 12, p.3323-3330, 2012.

GAMA, A.S.M; SECOLI, S.R. Automedicação em estudantes de enfermagem do Estado do Amazonas-Brasil. Revista Gaúcha de Enfermagem, v. 38, n. 1, 2017.

GIL AC. Métodos e técnicas de pesquisa social. São Paulo: Atlas; 2006.

Instituto Brasileiro de Geografia e Estatística. Brasil, Rondônia. Ouro Preto do Oeste. População no ultimo senso, 2017 [Online]. Disponível em: https://cidades.ibge.gov.br/brasil /ro/ouro-pretodo-oeste/panorama. Acesso em: 25 Mai, 2018.

ISRAEL, Ana Lins Martins. Atenção, dispensação e prescrição farmacêuticas em homeopatia. 2016.

JESUS, A.P.G.A.S.; YOSHIDA, N.C.P.; FREITAS, J.G.A.P. Prevalência da automedicação entre acadêmicos de Farmácia, Medicina, Enfermagem e Odontologia. Estudos. v. 40, n. 2, p. 151-164, 2013.

LOPES, W.F.L.et al. A prática da automedicação entre estudantes de uma instituição de ensino superior de Teresina-Pi. Revista Interdisciplinar. V.7, n.1, p. 17-24.2014.

MASNOON, $\mathrm{N}$ et al. What is polypharmacy? A systematic review of definitions. BMC geriatrics, v. 17, n. 1, p. 230, 2017

MARTINEZ, J.E. Estudo da automedicação para dor musculoesquelética entre estudantes dos cursos de enfermagem e medicina da Pontifícia Universidade Católica-São Paulo. Revista Brasileira de Reumatologia, v. 54, n. 2, p. 90-94, 2014.

MONTASTRUC, J.L. et al. Pharmacovigilance, risks and adverse effects of selfmedication. Thérapie, v. 71, n. 2, p. 257-262, 2016.

MONTASTRUC, J.L. et al. Pharmacovigilance, risks and adverse effects of selfmedication. Thérapie, v. 71, n. 2, p. 257-262, 2016.

MOURA, E.C; GOMES, R; PEREIRA, G.M.C. Percepções sobre a saúde dos homens numa perspectiva relacional de gênero, Brasil, 2014. Ciência Saúde Coletiva. V. 22, p. 291-300, 2017.

RAWLE, Mark James et al. The prevalence and determinants of polypharmacy at age 69: a British birth cohort study. BMC geriatrics, v. 18, n. 1, p. 118, 2018.

RIBEIRO, Maria Ângela; HEINECK, Isabela. Estoque domiciliar de medicamentos na comunidade ibiaense acompanhada pelo Programa Saúde da Família, em Ibiá-MG, Brasil. Saúde e Sociedade, v. 19, p. 653-663, 2010.

SANTOS, G.E.O. Cálculo amostral: calculadora on-line. Disponível em: http://www.calculoamostral.vai.la. Acesso em 01 de agosto de 2016. 
SCHMID, B. BERNAL, R. SILVA, N. N. A automedicação em adultos de baixa renda no município de São Paulo. Revista Saúde Pública. v. 44, n. 6, 2010;

SILVA, L. S. F. et al. Automedicação em acadêmicos de cursos de graduação da área da saúde de uma universidade privada do Sul do Estado de Minas Gerais. Odontol. Clín.-Cient., Recife, v. 10, n.1, p. 57 - 63, 2011.

SILVA, R.C.G.et al. Automedicação em acadêmicos do curso de medicina. Medicina (Ribeirão Preto). V. 45, n.1, p. 5-11,2012.

SILVA, I. M. et al. Self-medication in adolescence: a challenge to health education. Ciencia \& saude coletiva, v. 16, p. 1651-1660, 2011.

SILVA, W.C; SALVI, J.O. Medicamentos psicotrópicos, ansiedade e depressão em professores do município de cujubim - Rondônia. Brazilian Journal of Surgery and Clinical Research - BJSCR, Vol.21,n.2,pp.47-53 (Dez 2017 - Fev 2018).

TASIC, D.; MLADENOVSKA, D.; TASIC, L. Php33 utility of media on health promotion and selfmedication. Value in Health, v. 8, n. 3, p. 292, 2005.

WHO. World Health Organization. Medicines: rational use of medicines. Suécia, 2010. Disponível em: <http://www.who.int/mediacentre/ factsheets/fs338/en/>. Acesso em 02 ago. 2016.

WHO. World Health Organization. Journal of Rational Use of Medicine. Sudan, 2014.

Disponível em: < http://apps.who.int/medicinedocs/documents/s22205en/s22205en.pdf>. Acesso em 01 jun. 2018.

WHO. The Role of the Pharmacist in Self-Care and Self-Medication. Holanda, 1998. Disponível em: <http://apps.who.int/medicinedocs/en/d/Jwhozip32e/4.2.html>. Acesso em 01 jun. 2018. 